\title{
Regional differences in practice patterns and associated outcomes for upper tract urothelial carcinoma in Canada
}

\author{
Michael Metcalfe, MD, FRCSC,; Wassim Kassouf, MD, FRCSC, ${ }^{\dagger}$ Ricardo Rendon, MD, FRCSC, ${ }^{\ddagger}$ David Bell, MD, \\ FRCSC,; Jonathan Izawa, MD, FRCSC,; Joseph Chin, OOnt, MD, FRCSC; $;$ Anil Kapoor, MD, FRCSC;: Edward \\ Matsumoto, MD, FRCSC,: Jean-Baptiste Lattouf, MD, FRCSC, $;$ Fred Saad, MD, FRCSC,; Louis Lacombe, MD,

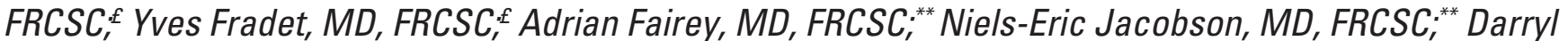 \\ Drachenberg, MD, FRCSC;, ;t llias Cagiannos, MD, FRCSC;, Alan So, MD, FRCSC; Peter Black, MD, FRCSC*
}

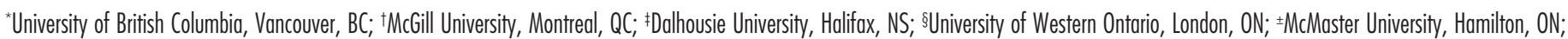
*University of Montreal, Montreal, QC; £ ¿aval University, Quebec, QC; ; University of Alberta, Edmonton, AB; ${ }^{+}$University of Manitoba, Winnipeg, MB; 执University of Ottawa, Ottawa, ON

See related article on page 463.

Cite as: Can Urol Assoc J 2012;6(6):455-62. http://dx.doi.org/10.5489/cuaj.12146

\section{Abstract}

Introduction: We delineated Canadian regional differences in practice patterns in the management of upper tract urothelial carcinoma (UTUC) after nephroureterectomy and relate these to patient outcomes. Methods: A database was created with 1029 patients undergoing radical nephroureterectomy for UTUC between 1994 and 2009 at 10 Canadian centres. Demographic, clinical and pathological variables were collected from chart review. Practice pattern variables were defined as: open versus laparoscopic nephroureterectomy, management strategy for the distal ureter, performance of lymphadenectomy and administration of chemotherapy and/or radiation therapy. The outcome measures were overall (OS), disease-specific (DSS) and recurrence-free survival (RFS). The centres were divided into three regions (West, Central, East). Cox proportional multivariable linear regression analysis was used to determine the association between regional differences in practice patterns and clinical outcomes. Results: There was a significant difference in practice patterns between regions within Canada for: time from diagnosis to surgery ( $p=0.001$ ), type of surgery (open vs. laparoscopic, $p<0.01)$ and method of management of the distal ureter $(p=0.001)$. As well, there were significant differences in survival between regions across Canada: 5-year OS (West 70\%, Central $81 \%$ and East $62 \%, p<0.0001$ ) and DSS (West $=79 \%$, Central $=85 \%$, East $=75 \%, p=0.007$ ) were significantly different, but there was no difference in RFS (West $47 \%$, Central $48 \%$, East $46 \%, p=0.88$ ). Multivariable linear regression analysis demonstrated that the differences in survival were independent of region OS $(p=0.78)$, DSS $(p=0.30)$ or RFS $(p=0.43)$. Conclusion: There is significant disparity in practice patterns between regions within Canada, but these do not appear to have an effect on survival. We believe that the variability in practice is a reflection of the lack of standardized treatments for UTUC and underlines the need for multi-institutional studies in this disease.

\section{Introduction}

Upper tract urothelial carcinoma (UTUC) is rare and comprises only $5 \%$ of urothelial cancers. ${ }^{1}$ This low incidence makes it relatively difficult to study and, as a result, much of our understanding of its natural history and pathology is extrapolated from bladder cancer. ${ }^{2}$ Currently, the gold standard treatment for UTUC is nephroureterectomy with excision of the bladder cuff. However, many aspects of the diagnosis and treatment of UTUC, such as the management of the distal ureter and bladder cuff, are specific to its anatomic location in the upper tract. The current evidence to address many of these management questions is derived from single or multi-institutional retrospective series. In this context, we wanted to study the current practice patterns across Canada in the management of UTUC.

The European Association of Urology (EAU) and the National Comprehensive Cancer Network (NCCN) have developed guidelines for the diagnosis and treatment of UTUC. ${ }^{2,3}$ These sources are consistent with each other on most issues, but they avoid concrete recommendations on many important questions. This is due mostly to the lack of quality evidence to support such recommendations. It would appear from multiple retrospective series, for example, that laparoscopic nephroureterectomy is equivalent to open nephroureterectomy, but this has not been evaluated prospectively. ${ }^{4-7}$ The most recent EAU guidelines state that there is limited data for laparoscopic versus open nephroureterectomy (grade B evidence), lymphadenectomy (Grade C evidence), and technique of bladder cuff excision (Grade $\mathrm{C}$ evidence). Other critical questions, such as the use of perioperative chemotherapy, have even less evidence.

We hypothesize that there is considerable variability in the management of UTUC primarily by nephroureterectomy across Canada and that this has a direct effect on patient outcome. We aim to define regional differences in practice patterns across Canada and relate these to patient survival. 
Metcalfe et al.

\section{Methods}

\section{Canadian Upper Tract Collaboration}

The Canadian Upper Tract Collaboration (CUTC) includes 10 Canadian centres. Each centre performed a chart review for predefined demographic, clinical and pathologic variables in all patients who underwent nephroureterectomy from 1990 to 2010. Participating sites included: University of British Columbia, University of Alberta, University of Winnipeg, University of Western Ontario, McMaster University, University of Ottawa, University of Montreal, McGill University, Laval University and Dalhousie University. All participating sites obtained necessary institutional data use approval prior to their involvement in the CUTC.

\section{Study design}

This is a retrospective study cohort of a multi-institutional database. The patient cohort was divided into three geographic regions: (1) West (British Columbia, Alberta and Manitoba); (2) Central (Ontario), and (3) East (Quebec and Maritime Provinces). Practice patterns were defined as: time from diagnosis to surgery, open versus laparoscopic nephroureterectomy, method of management of the distal ureter, performance of lymphadenectomy, use of neo-adjuvant and adjuvant chemotherapy, and use of radiation therapy. Baseline demographic, clinical and pathological parameters were collected. Demographic variables included age, gender, race and body mass index (BMI). Clinical variables included smoking history (never, quit and current), previous abdominal radiation exposure, presence of hydronephrosis, presence of symptoms (local vs. systemic), and a prior history of urothelial cancer (with associated stage, grade and treatment parameters). Pathological variables included

Table 1. Baseline characteristics and practice patterns for patients who underwent nephroureterectomy for UTUC in Canada, divided by geographical region

\begin{tabular}{|c|c|c|c|c|c|}
\hline & & Central & East & West & $p$ value \\
\hline \multicolumn{6}{|l|}{ Demographic characteristics } \\
\hline Total number & & 252 & 488 & 289 & \\
\hline Age & Mean & 70.2 (SD 10.5) & 69.8 (SD 10.4) & $69.1(11.2)$ & 0.231 \\
\hline \multirow{2}{*}{ Gender } & Male & $159 / 252(63 \%)$ & $320 / 488(65 \%)$ & $174 / 288(60 \%)$ & \multirow{2}{*}{0.340} \\
\hline & Female & $93 / 252(36.9 \%)$ & $168 / 488(34.4 \%)$ & $114 / 288(40 \%)$ & \\
\hline Past smoking history & & $183 / 285(78 \%)$ & $298 / 443(67 \%)$ & $165 / 247(67 \%)$ & 0.870 \\
\hline Body mass index $\left(\mathrm{mg} / \mathrm{m}^{2}\right)$ & Mean & 28.9 (SD 8.6) & 26.8 (SD 5.4) & 27.1 (SD 5.4) & 0.008 \\
\hline \multicolumn{6}{|l|}{ Clinical characteristics } \\
\hline History of abdominal radiation & & $8 / 249(3.2 \%)$ & $30 / 352(8.5 \%)$ & $42 / 282(15 \%)$ & $<0.001$ \\
\hline Presence of hydronephrosis & & $149 / 235(63.4 \%)$ & $152 / 349(42.9 \%)$ & $151 / 285(53 \%)$ & $<0.001$ \\
\hline \multirow{3}{*}{ Presence of symptoms } & None & $35 / 247(14 \%)$ & $44 / 455(10 \%)$ & $27 / 278(10 \%)$ & \multirow{3}{*}{0.083} \\
\hline & Local & $205 / 247(83 \%)$ & $385 / 455(85 \%)$ & $231 / 278(83 \%)$ & \\
\hline & Systemic & $7 / 247(3 \%)$ & $26 / 455(6 \%)$ & $20 / 278(7 \%)$ & \\
\hline History of bladder cancer & & $69 / 249(28 \%)$ & $124 / 479(26 \%)$ & $75 / 283(26.5 \%)$ & 0.870 \\
\hline History of UTUC & & $18 / 247(7.29 \%)$ & $28 / 353(7.9 \%)$ & $33 / 282(11.7 \%)$ & 0.142 \\
\hline \multirow{4}{*}{ Management of prior UTUC } & Endoscopic & $11 / 16(69 \%)$ & $13 / 27(48 \%)$ & $23 / 33(70 \%)$ & \multirow{4}{*}{0.177} \\
\hline & Percutaneous & $1 / 16(6 \%)$ & $5 / 27(19 \%)$ & $0 / 33(0 \%)$ & \\
\hline & Distal & $3 / 16(19 \%)$ & $6 / 27(22 \%)$ & $5 / 33(15 \%)$ & \\
\hline & Segmental & $1 / 16(6 \%)$ & $3 / 27(11 \%)$ & $5 / 33(15 \%)$ & \\
\hline \multirow{2}{*}{ Grade of highest previous cancer } & Low & $28 / 54(52 \%)$ & $36 / 101(35.6 \%)$ & $37 / 71(52 \%)$ & \multirow{2}{*}{0.049} \\
\hline & High & $26 / 54(48 \%)$ & $65 / 101(64.4 \%)$ & $34 / 71(48 \%)$ & \\
\hline \multirow{2}{*}{ Stage of highest previous cancer } & $\mathrm{Ta}$, Tis and $\mathrm{T} 1$ & $41 / 47(87 \%)$ & $71 / 85$ (83.5\%) & $50 / 67(75 \%)$ & \multirow{2}{*}{0.188} \\
\hline & T2, T3 or T4 & $6 / 47(13 \%)$ & $14 / 85(16.5 \%)$ & $17 / 67(25 \%$ & \\
\hline \multicolumn{6}{|l|}{ Pathological characteristics } \\
\hline Presence of CIS & & $18 / 58(31 \%)$ & $13 / 100(13 \%)$ & $20 / 66(30 \%)$ & 0.007 \\
\hline \multirow{3}{*}{ Tumour location } & Renal Pelvis & $129 / 245(53 \%)$ & $272 / 470(58 \%)$ & $137 / 286(48 \%)$ & \multirow{3}{*}{$<0.001$} \\
\hline & Prox. ureter & $52 / 245(21 \%)$ & $109 / 470(23 \%)$ & $89 / 286(31 \%)$ & \\
\hline & Pelvis + ureter & $64 / 245(26 \%)$ & $89 / 470(19 \%)$ & $60 / 286(21 \%)$ & \\
\hline Presence of multifocality & & $94 / 246(38 \%)$ & $63 / 340(19 \%)$ & $82 / 285(29 \%)$ & $<0.001$ \\
\hline
\end{tabular}


tumour location within the renal pelvis or ureter, stage, grade, multifocality, carcinoma in situ $(\mathrm{CIS})$, the presence of lymphovascular invasion, architecture (papillary vs. sessile), lymph node status, and surgical resection margin status. Outcome measures were overall survival (OS), diseasespecific survival (DSS) and recurrence-free survival (RFS) at 5 years after nephroureterectomy. All surgical pathology was examined according to usual clinical practice at each individual institution.

\section{Statistical analysis}

Data were analyzed using Statistical Analysis Software version 9.1.3 (SAS Institute, Cary, NC). The Fisher's exact and chi-squared tests were used to evaluate differences between geographical regions. Univariable and multivariable cox proportional regression analyses were performed to determine the association between practice patterns and clinical outcomes. All significant or borderline significant variables from the univariable analysis were included in the multivariable model. A two-sided $p$ value $<0.05$ defined statistical significance, and a $p$ value $<0.2$ defined borderline significance. The Kaplan-Meier method was used to estimate survival functions; differences were assessed with the Log-rank statistic.

\section{Results}

\section{Baseline characteristics}

The CUTC included data on 1029 patients who underwent nephroureterectomy from 10 different Canadian centres. There were 289 patients from the West, 252 patients from Central Canada and 488 patients from the East. The mean follow-up was 2.2 years (range: 0.6-5.0 years) for all patients. It was 1.8 years in Western Canada (range: 0.54.9), 2.1 years in Central Canada (range: 0.7-4.4), and 2.0 in Eastern Canada (range: 0.7-4.8). Demographics were similar between the three study regions (Table 1). However, patients from Central Canada had a statistically significantly higher BMI. Clinical parameters that carried statistical significance

\begin{tabular}{|c|c|c|c|c|c|}
\hline Pathological characteristics (cont'd) & & Central & East & West & $p$ value \\
\hline \multirow{3}{*}{ Architecture } & Papillary & $215 / 251(86 \%)$ & $286 / 488(59 \%)$ & $240 / 288(83 \%)$ & \multirow{3}{*}{$<0.001$} \\
\hline & Sessile & 9/251 (4\%) & $11 / 488(2 \%)$ & $5 / 288(1.7 \%)$ & \\
\hline & Other & $27 / 251(10 \%)$ & $191 / 488(39 \%)$ & $43 / 288(15 \%)$ & \\
\hline Presence of lymphvascular invasion & & $38 / 252(15 \%)$ & $79 / 488(16 \%)$ & $50 / 289(17 \%)$ & $<0.001$ \\
\hline Presence of positive surgical margins & & $20 / 244(8 \%)$ & $10.3 / 443(10.3)$ & $34 / 281(12 \%)$ & 0.341 \\
\hline \multirow{3}{*}{ Presence of positive lymph nodes } & No & $60 / 252(24 \%)$ & $59 / 488(12 \%)$ & $67 / 289(23 \%)$ & \multirow{3}{*}{$<0.001$} \\
\hline & N1 & $19 / 252(8 \%)$ & $38 / 488(7.8 \%)$ & $21 / 289(7.3 \%)$ & \\
\hline & $\mathrm{Nx}$ & $173 / 252(69 \%)$ & $391 / 488(80 \%)$ & $201 / 289(70 \%)$ & \\
\hline \multirow{4}{*}{ Stage } & T1 & $130 / 235(55 \%)$ & $204 / 440(46 \%)$ & $129 / 246(52 \%)$ & \multirow{4}{*}{$<0.144$} \\
\hline & T2 & $43 / 235(18 \%)$ & $81 / 440(18 \%)$ & $36 / 246(14 \%)$ & \\
\hline & T3 & $48 / 235(20 \%)$ & $131 / 440(30 \%)$ & $65 / 246(26 \%)$ & \\
\hline & T4 & $14 / 235(5 \%)$ & $24 / 440(5 \%)$ & $16 / 246(6.5 \%)$ & \\
\hline \multirow{2}{*}{ Grade } & Low & $99 / 243(40 \%)$ & $123 / 477(26 \%)$ & $90 / 281(32 \%)$ & \multirow{2}{*}{$<0.001$} \\
\hline & High & $144 / 243(59 \%)$ & $453 / 477(74 \%)$ & $191 / 281(68 \%)$ & \\
\hline \multicolumn{6}{|l|}{ Practice pattern } \\
\hline Time from diagnosis to surgery (days) & & $92.8(92.4)$ & $36.8(57)$ & $68.4(104)$ & $<0.001$ \\
\hline \multirow{2}{*}{ Surgery type } & Open & $91 / 252(36 \%)$ & $197 / 309(64 \%)$ & $115 / 288(40 \%)$ & \multirow{2}{*}{$<0.001$} \\
\hline & Laparoscopic & $161 / 252(64 \%)$ & $112 / 309(36 \%)$ & $173 / 288(60 \%)$ & \\
\hline \multirow{4}{*}{ Management of distal ureter } & Extravesical & $67 / 252(27 \%)$ & $105 / 299(35 \%)$ & $144 / 284(51 \%)$ & \multirow{4}{*}{$<0.001$} \\
\hline & Internal/external & $103 / 252(41 \%)$ & $184 / 299(62 \%)$ & $119 / 284(42 \%)$ & \\
\hline & Endoscopic & $77 / 252(31 \%)$ & $8 / 299(3 \%)$ & $13 / 284(5 \%)$ & \\
\hline & Other & $5 / 252(2 \%)$ & $2 / 299(1 \%)$ & $8 / 284(3 \%)$ & \\
\hline Lymphadenectomy & & $79 / 251(31 \%)$ & $99 / 481(21 \%)$ & $98 / 283(35 \%)$ & $<0.001$ \\
\hline Neoadjuvant chemotherapy & & $3 / 248(1.2 \%)$ & $13 / 353(3.7 \%)$ & $15 / 289(5.2 \%)$ & 0.042 \\
\hline Adjuvant chemotherapy & & $21 / 249(8.4 \%)$ & $53 / 479(11 \%)$ & $38 / 289(13 \%)$ & 0.219 \\
\hline Salvage chemotherapy & & $32 / 234(14 \%)$ & $18 / 352(5.1 \%)$ & $25 / 289(8.7 \%)$ & $<0.001$ \\
\hline Salvage radiation therapy & & $15 / 241(6.2 \%)$ & $31 / 352(9 \%)$ & $23 / 289(8.0 \%)$ & 0.513 \\
\hline
\end{tabular}


Metcalfe et al.

between regions were: previous abdominal radiation, the presence of hydronephrosis and highest grade of any prior tumour. Pathological parameters that were statistically different between regions included: presence of CIS, multifocality, tumour location, architecture, lymphovascular invasion, tumour node status and tumour grade.

Practice patterns varied significantly across Canada. Differences in the time from diagnosis to surgery, proportion of open versus laparoscopic nephroureterectomy, method of distal ureteral management, performance of lymphadenectomy and use of chemotherapy were statistically significant between regions.

\section{Clinical outcomes stratified by geographic region}

We used the Kaplan-Meier method OS (Fig. 1), DSS (Fig. 2) and RFS estimates (Fig. 3), stratified by geographical region. The predicted 5-year OS (West 69.6\%, Central 80.6\% and East $62.3 \%, p<0.0001$, log rank statistic $=0.014)$ and DSS (West 79.2\%, Central 84.9\%, East 75.0\%, $p=0.007$, log rank $=0.13$ ) differed significantly by region, but the RFS (West $46.7 \%$, Central $47.6 \%$, East $45.7 \%, p=0.88 \log$ rank=0.40) did not.

\section{Association between geographic region and clinical outcomes}

Table 2 presents the results of the univariable analysis examining predictors of clinical outcomes. Geographic region was associated with 5 -year OS $(p<0.001)$ and DSS $(p=0.007)$, but not with RFS ( $p=0.88)$. Table 3 presents the results of the multivariable analysis examining predictors of clinical outcomes. Geographic region was included in the multivariable analysis, but it did not make the final model. It was not independently associated with OS $(p=0.78)$, DSS $(p=0.30)$ or RFS $(p=0.43)$.

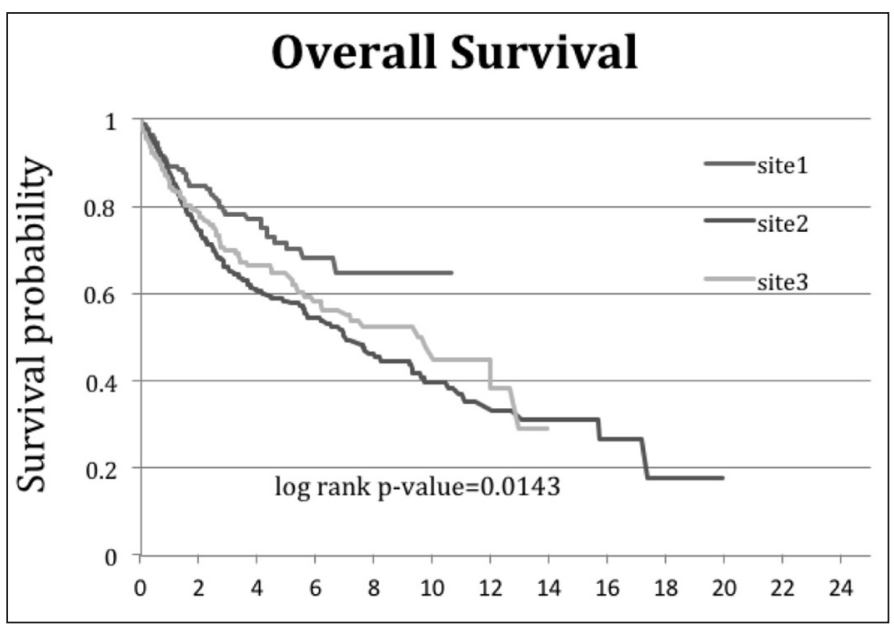

Fig. 1. Kaplan-Meier curve for overall survival stratified by geographic region.

\section{Discussion}

In this study, we analyzed practice patterns across Canada with respect to the management of UTUC and correlated these to clinical outcomes. We found that practice patterns varied considerably by region, but there was no significant difference in outcome after controlling for demographic, clinical and pathological variables. Baseline variability for clinical and pathological parameters was surprising, but can likely be explained by cultural, genetic, and socio-economic variability, in addition to referral patterns to the respective centres. The differences in pathologic parameters could also be due to variability at the individual centres for determining the indication for nephroureterectomy. The regional differences in practice patterns pertained particularly to surgical wait times, laparoscopic versus open nephroureterectomy, management of the distal ureter and the use of chemotherapy and radiation therapy.

OS and DSS, but not RFS, correlated with geographic region. However, when geographic region was included in the multivariable analysis, it did not independently predict survival. Patients in central Canada had the longest 5-year OS and DSS, despite some adverse characteristics, such as increased BMI and smoking history as well as more frequent hydronephrosis and more delays in treatment. Other diseaseand treatment-related factors appear to have compensated for this, including more frequent low-grade disease with papillary architecture and more frequent $\mathrm{NO}$ status. The time from diagnosis to surgery demonstrated remarkable variance across Canada. Patients in Central Canada waited 2.5 times longer (92.8 days) and patients in Western Canada 1.9 times (68 days) longer than patients in Eastern Canada (36.8 days) to reach the operating theatre $(p=0.001)$. However, wait time did not correlate with OS (hazard ratio $[H R]=0.999$ ), DSS (HR=0.998) or RFS (HR=0.998). We know from studies in urothelial carcinoma of the bladder that delays in radical cystectomy greater than 12 weeks lead to decreased

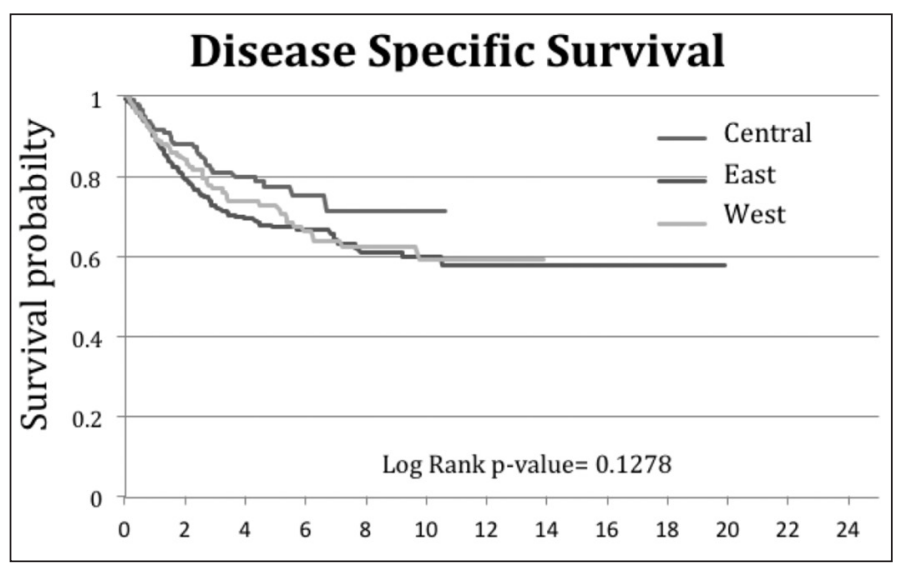

Fig. 2. Kaplan-Meier curve for disease specific survival stratified by geographic region. 
UTUC in Canada: regional differences

\begin{tabular}{|c|c|c|c|c|c|c|c|c|c|c|}
\hline \multirow[t]{2}{*}{ Demographic variables } & \multirow[b]{2}{*}{ West } & \multicolumn{2}{|c|}{ 5-year OS } & \multirow[t]{2}{*}{$p$ value } & \multicolumn{2}{|c|}{ 5-year DSS } & \multirow[t]{2}{*}{$p$ value } & \multicolumn{2}{|c|}{ 5-year RFS } & \multirow[t]{2}{*}{$p$ value } \\
\hline & & $201 / 289$ & $69.6 \%$ & & $229 / 289$ & $79.2 \%$ & & $135 / 289$ & $46.7 \%$ & \\
\hline \multirow[t]{2}{*}{ Site } & Central & $203 / 252$ & $80.6 \%$ & $<0.001$ & $214 / 252$ & $84.9 \%$ & 0.879 & $120 / 252$ & $47.6 \%$ & 0.879 \\
\hline & East & $304 / 488$ & $62.3 \%$ & & $366 / 488$ & $75.0 \%$ & & $223 / 488$ & $45.7 \%$ & \\
\hline \multirow{2}{*}{ Sex } & Male & $444 / 653$ & $68.0 \%$ & \multirow{2}{*}{0.476} & $507 / 653$ & $77.6 \%$ & \multirow{2}{*}{0.323} & $308 / 653$ & $47.2 \%$ & \multirow{2}{*}{0.516} \\
\hline & Female & $263 / 375$ & $70.1 \%$ & & $301 / 375$ & $80.2 \%$ & & $169 / 375$ & $45.1 \%$ & \\
\hline \multirow{2}{*}{ Smoking history } & Non-Smoker & $213 / 279$ & $76.3 \%$ & \multirow{2}{*}{0.005} & $236 / 279$ & $84.6 \%$ & \multirow{2}{*}{0.010} & $144 / 279$ & $51.6 \%$ & \multirow{2}{*}{0.029} \\
\hline & Smoker & $433 / 646$ & $67.0 \%$ & & $498 / 646$ & $77.1 \%$ & & $283 / 646$ & $43.2 \%$ & \\
\hline \multicolumn{11}{|l|}{ Clinical variables } \\
\hline \multirow{2}{*}{ Hydronephrosis } & None & $213 / 279$ & $76.3 \%$ & \multirow{2}{*}{0.441} & $347 / 422$ & $82.0 \%$ & \multirow{2}{*}{0.080} & $209 / 422$ & $49.5 \%$ & \multirow{2}{*}{0.068} \\
\hline & Hydronephrosis & $433 / 646$ & $67.0 \%$ & & $349 / 452$ & $78.2 \%$ & & $196 / 452$ & $43.4 \%$ & \\
\hline Previous abdominal & None & $575 / 803$ & $71.6 \%$ & 0590 & $643 / 803$ & $80.1 \%$ & 0.417 & $380 / 803$ & $47.3 \%$ & \\
\hline radiation & Prior RT & $56 / 79$ & $70.8 \%$ & 0.590 & $61 / 80$ & $76.2 \%$ & 0.417 & $32 / 80$ & $40.0 \%$ & 0.211 \\
\hline Provious UTUC & None & $574 / 803$ & $71.4 \%$ & 0911 & $638 / 803$ & $79.5 \%$ & 0387 & $375 / 803$ & $46.7 \%$ & 0000 \\
\hline Previous uiuc & Prior & $56 / 79$ & $70.8 \%$ & 0.911 & $66 / 79$ & $83.5 \%$ & $0.38 \%$ & $37 / 79$ & $46.8 \%$ & 0.982 \\
\hline History of bladder & None & $473 / 634$ & $74.6 \%$ & 0006 & $598 / 743$ & $80.5 \%$ & 0020 & $360 / 743$ & $48.5 \%$ & $=0020$ \\
\hline cancer & Yes & $170 / 268$ & $63.4 \%$ & 0.000 & $198 / 268$ & $73.9 \%$ & $0.0 \angle 4$ & $108 / 268$ & $60.3 \%$ & $<0.0<2$ \\
\hline & None & $86 / 106$ & $81.1 \%$ & & $90 / 106$ & $84.9 \%$ & & $61 / 106$ & $57.5 \%$ & \\
\hline Symptoms from UTCC & Local & $570 / 821$ & $69.4 \%$ & 0.027 & $654 / 821$ & $79.7 \%$ & 0.083 & $381 / 821$ & $46.4 \%$ & 0.091 \\
\hline & Systemic & $34 / 53$ & $64.2 \%$ & & $37 / 53$ & $69.8 \%$ & & $24 / 53$ & $45.3 \%$ & \\
\hline Pathological variables & & & & & & & & & & \\
\hline & Renal pelvis & $394 / 538$ & $73.2 \%$ & & $446 / 538$ & $82.8 \%$ & & $285 / 538$ & $53.0 \%$ & \\
\hline Tumour location & Ureter & $174 / 250$ & $69.6 \%$ & 0.001 & $196 / 250$ & $78.4 \%$ & $<0.001$ & $119 / 250$ & $47.6 \%$ & $<0.001$ \\
\hline & Both & $126 / 213$ & $59.2 \%$ & & $146 / 213$ & $68.5 \%$ & & $66 / 213$ & $31.0 \%$ & \\
\hline Multifocal diceace & None & $473 / 634$ & $74.6 \%$ & 0006 & $598 / 743$ & $81.5 \%$ & 0024 & $302 / 634$ & $47.6 \%$ & $=0001$ \\
\hline Multifocal disease & Yes & $156 / 239$ & $65.2 \%$ & 0.006 & $198 / 268$ & $73.9 \%$ & 0.024 & $78 / 239$ & $32.6 \%$ & $<0.001$ \\
\hline & T1 & $357 / 463$ & $75.8 \%$ & & $403 / 463$ & $87.0 \%$ & & $256 / 463$ & $55.3 \%$ & \\
\hline Tumour pathology & T2 & $114 / 160$ & $71.3 \%$ & n001 & $128 / 160$ & $80.0 \%$ & $n 001+$ & $72 / 160$ & $45.0 \%$ & 0001 \\
\hline stage & T3 & $140 / 244$ & $57.4 \%$ & .007 & $169 / 244$ & $69.3 \%$ & $<0.001$ & $153 / 244$ & $37.3 \%$ & $<0.001$ \\
\hline & T4 & $26 / 54$ & $48.1 \%$ & & $31 / 54$ & $57.4 \%$ & & $16 / 54$ & $29.6 \%$ & \\
\hline Tumour arade & Low & $251 / 312$ & $80.4 \%$ & $<0001$ & $274 / 312$ & $87.8 \%$ & $<0001$ & $171 / 312$ & $54.8 \%$ & 00006 \\
\hline tumbur grade & High & $442 / 689$ & $64.1 \%$ & $<0.001$ & $516 / 689$ & $74.9 \%$ & $<0.001$ & $392 / 689$ & $43.1 \%$ & 0.0000 \\
\hline Lymphovascular & Absent & $460 / 615$ & $74.8 \%$ & 1 & $515 / 615$ & $83.7 \%$ & $=0001$ & $304 / 615$ & $49.4 \%$ & $=0001$ \\
\hline invasion & Present & 149/247 & $60.3 \%$ & $<1$ & 107/167 & $64.1 \%$ & $<0.001$ & $118 / 247$ & $47.7 \%$ & $<0.001$ \\
\hline Architecture & Papillary & $554 / 741$ & $74.7 \%$ & $<0,001$ & $615 / 741$ & $83.0 \%$ & $<0001$ & $364 / 741$ & $49.1 \%$ & $<0006$ \\
\hline Arcnitecture & Sessile & $11 / 25$ & $44.0 \%$ & & $13 / 25$ & $52.0 \%$ & & $6 / 25$ & $24.0 \%$ & \\
\hline CIS & Absent & $490 / 650$ & $75.3 \%$ & 0002 & $542 / 650$ & $83.4 \%$ & $000 ?$ & $341 / 650$ & $52.5 \%$ & $<0001$ \\
\hline Clo & Present & 139/215 & $64.6 \%$ & .002 & $159 / 215$ & $74.0 \%$ & 0.002 & $68 / 215$ & $31.6 \%$ & $<0.001$ \\
\hline Surgical marains & Negative & $620 / 868$ & $71.4 \%$ & $<0.001$ & $704 / 868$ & $81.1 \%$ & $<0.001$ & $426 / 868$ & $49.1 \%$ & $<0.001$ \\
\hline Surgical margens & Positive & $47 / 100$ & $47.0 \%$ & & $59 / 100$ & $59.0 \%$ & & $21 / 100$ & $21.0 \%$ & \\
\hline & No & $132 / 186$ & $71.0 \%$ & & $148 / 186$ & $79.6 \%$ & & $87 / 186$ & $46.8 \%$ & \\
\hline Lymph node status & N1 & $33 / 78$ & $42.3 \%$ & $<0.001$ & $39 / 78$ & $50.0 \%$ & $<0.001$ & $15 / 78$ & $19.2 \%$ & $<0.001$ \\
\hline & $\mathrm{Nx}$ & $543 / 765$ & $71.0 \%$ & & $622 / 765$ & $81.3 \%$ & & $376 / 765$ & $49.2 \%$ & \\
\hline
\end{tabular}

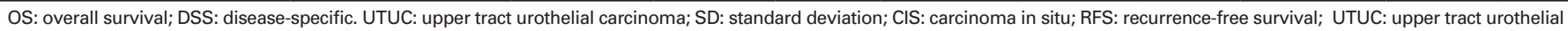
carcinoma; SD: standard deviation; CIS: carcinoma in situ.

survival. ${ }^{8}$ There is limited data for such a relationship in UTUC. One study showed an increased risk of upstaging with delays in nephroureterectomy greater than 45 days, however there was no correlation with survival. ${ }^{9} \mathrm{~A}$ small retrospective review demonstrated that, in carefully selected patients, including some after neoadjuvant chemotherapy, a prolonged surgical wait time has no effect on survival. ${ }^{10}$ The difference with UTUC is that tumours are more likely low grade and more often non-muscle invasive, whereas in the bladder, tumours are almost all high grade, and many are muscle invasive. Wait time may not be as critical for the lower risk tumours in the upper tract. 
Metcalfe et al.

\begin{tabular}{|c|c|c|c|c|c|c|c|c|c|c|}
\hline \multicolumn{2}{|l|}{ Practice pattern } & \multicolumn{2}{|c|}{ 5-year OS } & \multirow{3}{*}{$\begin{array}{l}p \text { v value } \\
<0.001\end{array}$} & \multicolumn{2}{|c|}{ 5-year DSS } & \multirow{3}{*}{$\begin{array}{r}\boldsymbol{p} \text { value } \\
<0.001\end{array}$} & \multicolumn{2}{|c|}{ 5-year RFS } & \multirow{3}{*}{$\begin{array}{r}\boldsymbol{p} \text { value } \\
0.0191\end{array}$} \\
\hline \multirow{2}{*}{ Surgery type } & Open & $265 / 403$ & $66.0 \%$ & & $310 / 403$ & $80.5 \%$ & & $179 / 403$ & $44.4 \%$ & \\
\hline & Laparoscopic & $365 / 446$ & $82.1 \%$ & & $387 / 446$ & $73.9 \%$ & & $234 / 446$ & $62.5 \%$ & \\
\hline \multirow{2}{*}{ Lymphadenectomy } & None & $525 / 739$ & $71.0 \%$ & \multirow{2}{*}{0.019} & $600 / 739$ & $82.2 \%$ & \multirow{2}{*}{0.8431} & $362 / 739$ & $49.0 \%$ & \multirow{2}{*}{0.0051} \\
\hline & Performed & $175 / 276$ & $63.4 \%$ & & $198 / 276$ & $71.7 \%$ & & $108 / 276$ & $39.1 \%$ & \\
\hline \multirow{3}{*}{$\begin{array}{l}\text { Distal ureter } \\
\text { management }\end{array}$} & Extravesical & $236 / 316$ & $74.7 \%$ & \multirow{3}{*}{0.179} & $263 / 316$ & $83.2 \%$ & \multirow{3}{*}{0.398} & $146 / 316$ & $46.2 \%$ & \multirow{3}{*}{0.0825} \\
\hline & Transvesical & $314 / 406$ & $77.3 \%$ & & $339 / 406$ & $83.5 \%$ & & $217 / 406$ & $53.4 \%$ & \\
\hline & Endocopic & $8 / 15$ & $53.3 \%$ & & $82 / 98$ & $83.4 \%$ & & $43 / 98$ & $43.8 \%$ & \\
\hline \multirow{2}{*}{$\begin{array}{l}\text { Neoadjuvant } \\
\text { chemotherapy }\end{array}$} & None & $617 / 859$ & $71.8 \%$ & \multirow{2}{*}{0.375} & $691 / 859$ & $80.5 \%$ & \multirow{2}{*}{0.030} & $406 / 859$ & $47.3 \%$ & \multirow{2}{*}{0.100} \\
\hline & Given & $20 / 31$ & $64.5 \%$ & & 20/31 & $64.5 \%$ & & $10 / 31$ & $32.3 \%$ & \\
\hline \multirow{2}{*}{$\begin{array}{l}\text { Adjuvant } \\
\text { chemotherapy }\end{array}$} & None & $631 / 905$ & $69.7 \%$ & \multirow{2}{*}{0.052} & $726 / 905$ & $80.2 \%$ & \multirow[t]{2}{*}{$<0.001$} & $453 / 905$ & $51.1 \%$ & \multirow{2}{*}{$<0.001$} \\
\hline & Given & $68 / 112$ & $60.7 \%$ & & $72 / 112$ & $64.3 \%$ & & $21 / 112$ & $78.7 \%$ & \\
\hline \multirow{2}{*}{ Salvage chemotherapy } & None & $591 / 800$ & $73.9 \%$ & \multirow{2}{*}{$<0.001$} & $664 / 800$ & $83.0 \%$ & \multirow{2}{*}{$<0.001$} & $410 / 800$ & $51.2 \%$ & \multirow{2}{*}{$<0.001$} \\
\hline & Given & $33 / 75$ & $44 \%$ & & $33 / 75$ & $54.0 \%$ & & $0 / 75$ & $0 \%$ & \\
\hline \multirow{2}{*}{ Salvage radiotherapy } & None & $612 / 813$ & $75.3 \%$ & \multirow{2}{*}{$<0.001$} & $685 / 813$ & $84.3 \%$ & \multirow{2}{*}{$<0.001$} & $414 / 813$ & $51.0 \%$ & \multirow{2}{*}{$<0.001$} \\
\hline & Given & $18 / 69$ & $26.1 \%$ & & $19 / 69$ & $27.5 \%$ & & & $0 \%$ & \\
\hline \multicolumn{2}{|c|}{ Time from diagnosis to surgery (days) } & $641 / 924$ & 64.6 & 0.0051 & 731/924 & 63.9 & 0.0018 & 431/924 & 66.06 & 0.026 \\
\hline
\end{tabular}

OS: overall survival; DSS: disease-specific. UTUC: upper tract urothelial carcinoma; SD: standard deviation; CIS: carcinoma in situ. survival; RFS: recurrence-free survival; UTUC: upper tract urothelial carcinoma; SD: standard deviation; CIS: carcinoma in situ.

The regional differences in wait times presumably result from local logistics related to access to imaging studies, operating room time and other such variables. We acknowledge that our data are taken from 10 tertiary care referral centres and this may have a large impact on wait times and may not be representative of community centres across Canada.

This study parallels a previous Canadian study on wait times for bladder cancer patients undergoing cystectomy. In a mix of community and academic centres, there was a large variability in wait times. ${ }^{11}$ Because Canadian centres were previously beyond the recommended threshold of the 40-day wait time, ${ }^{12,13}$ in 2006 the surgical wait times initiative (SWAT) was developed to expedite surgery for all genitourinary malignancies. ${ }^{14}$ The patients from the current study were treated between 1990 and 2009, which mostly pre-dates SWAT.

There were significant differences in the surgical techniques used across the country. The diminished use of laparoscopy in Eastern Canada (36\%) compared to western (60\%) and central (64\%) regions may reflect a delayed adoption of laparoscopy in general, or a hesitation to adopt this technique specifically for nephroureterectomy. We now know from our own collaboration ${ }^{15}$ and from other series ${ }^{6,16,17}$ that the oncologic control for laparoscopic and open nephroureterectomy appears to be the same, but this was previously debated. Interestingly, the rate of laparoscopic cases done in Canada in the 10-year study period was much higher than the number reported in the largest multi-institutional series published, where only $28 \% \%$ (270/1249) of patients from 9 centres underwent laparoscopic nephroureterectomy from 1987 to 2007 .
Regional differences in the management of the distal ureter and bladder cuff are not surprising, as there are numerous options for this part of the surgery, and there is little evidence to support one over another. There has also likely been some evolution in practice over the study period. As with laparoscopy, it is noteworthy that Canadian urologists are excising the distal ureter and bladder cuff more frequently than was reported by the largest multi-institutional series, in which $32.2 \%$ (403 of 1249) of patients underwent a bladder cuff excision.?

The regional differences in the management of the distal ureter and bladder cuff underline a common theme that is apparent throughout the management of patients with UTUC: poor standardization of practices. Highly variable practice patterns are likely a reflection primarily of the low level of evidence guiding practice for UTUC. Few prospective or randomized studies have been conducted in this

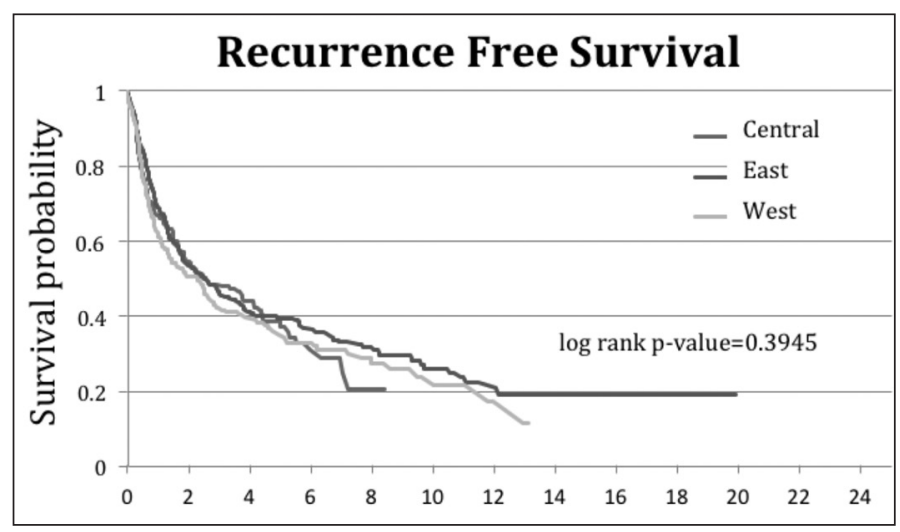

Fig. 3. Kaplan-Meier curve for recurrence free survival stratified by geographic region. 
UTUC in Canada: regional differences

\begin{tabular}{|c|c|c|c|c|c|c|c|c|c|}
\hline \multirow{2}{*}{ Variable } & \multicolumn{3}{|c|}{ OS } & \multicolumn{3}{|c|}{ DSS } & \multicolumn{3}{|c|}{ RFS } \\
\hline & HR & \multicolumn{2}{|c|}{$95 \% \mathrm{Cl}$} & \multirow[t]{2}{*}{ HR } & \multicolumn{2}{|c|}{$95 \% \mathrm{Cl}$} & \multirow[t]{2}{*}{ HR } & \multicolumn{2}{|c|}{$95 \% \mathrm{Cl}$} \\
\hline \multicolumn{8}{|l|}{ Region } & & \\
\hline Central & 1.0 & & & 1.0 & & & 1.0 & & \\
\hline East & 1.105 & 0.707 & 1.727 & 1.097 & 0.658 & 1.827 & 0.796 & 0.575 & 1.103 \\
\hline West & 1.124 & 0.744 & 1.698 & 0.892 & 0.540 & 1.473 & 0.726 & 0.519 & 1.016 \\
\hline \multicolumn{10}{|l|}{ Age } \\
\hline$<65$ & 1.0 & & & 1.0 & & & 1.0 & & \\
\hline$>65$ & 1.045 & 1.027 & 1.063 & 1.034 & 1.013 & 1.055 & 1.023 & 1.010 & 1.035 \\
\hline \multicolumn{10}{|l|}{ Symptoms } \\
\hline None & 1.0 & & & 1.0 & & & 1.0 & & \\
\hline Local/Systemic & - & - & - & 1.725 & 0.906 & 3.282 & 1.740 & 1.166 & 2.595 \\
\hline \multicolumn{10}{|l|}{ Surgical Approach } \\
\hline Open & 1.0 & & & 1.0 & & & 1.0 & & \\
\hline Laparoscopic & 0.866 & 0.618 & 1.270 & - & - & - & 1.142 & 1.085 & 1.839 \\
\hline \multicolumn{10}{|l|}{ Tumour location } \\
\hline pelvis & 1.0 & & & 1.0 & & & 1.0 & & \\
\hline ureter & 1.165 & 0.770 & 1.762 & 1.246 & 0.754 & 2.058 & - & - & - \\
\hline ureter and pelvis & 2.021 & 1.395 & 2.927 & 2.379 & 1.484 & 3.814 & 1.779 & 1.345 & 2.354 \\
\hline \multicolumn{10}{|l|}{ pT stage } \\
\hline 1 & 1.0 & & & 1.0 & & & 1.0 & - & - \\
\hline 2 & 0.987 & 0.606 & 1.606 & 1.145 & 0.634 & 2.069 & - & - & - \\
\hline 3 & 2.564 & 1.748 & 3.759 & 2.935 & 1.810 & 4.758 & - & - & - \\
\hline 4 & 4.326 & 2.427 & 7.714 & 3.660 & 1.723 & 7.777 & - & - & - \\
\hline Tumour grade & & & & & & & & & \\
\hline Low & 1.0 & & & 1.0 & & & $\begin{array}{c}1.0 \\
1395\end{array}$ & 1.059 & 1.839 \\
\hline High & 2.158 & 1.426 & 3.265 & 2.158 & 1.273 & 3.658 & & & \\
\hline Concomitant CIS & & & & & & & & & \\
\hline None & 1.0 & & & 1.0 & & & 1.0 & & \\
\hline Present & 0.819 & 0.566 & 1.184 & 0.847 & 0.547 & 1.311 & - & - & - \\
\hline History of bladder & & & & & & & & & \\
\hline None & 1.0 & & & 1.0 & & & 1.0 & & \\
\hline Yes & 1.313 & 0.946 & 1.822 & 1.367 & 0.910 & 2.052 & 1.124 & 0.845 & 1.496 \\
\hline Neoadjuvant chem & & & & & & & & & \\
\hline None & 1.0 & & & 1.0 & & & 1.0 & & \\
\hline Yes & - & - & - & 2.573 & 0.981 & 6.748 & 0.995 & 0.457 & 1.998 \\
\hline Adjuvant chemoth & & & & & & & & & \\
\hline None & 1.0 & & & 1.0 & & & 1.0 & & \\
\hline Yes & - & - & - & - & - & - & 1.559 & 1.089 & 2.231 \\
\hline Salvage chemothe & & & & & & & & & \\
\hline None & 1.0 & & & 1.0 & 1.0 & & 1.0 & & \\
\hline Yes & 1.187 & 0.719 & 1.960 & 1.744 & 1.021 & 2.978 & 2.341 & 1.601 & 3.424 \\
\hline Salvage radiation & & & & & & & & & \\
\hline None & 1.0 & & & 1.0 & & & 1.0 & & \\
\hline Yes & 3.586 & 2.272 & 5.662 & 4.018 & 2.419 & 6.675 & 2.410 & 1.661 & 3.497 \\
\hline Surgical margins & & & & & & & & & \\
\hline Negative & 1.0 & & & 1.0 & & & 1.0 & & \\
\hline Positive & - & - & - & 1.342 & 0.790 & 2.279 & 1.428 & 0.995 & 2.050 \\
\hline Smoking history & & & & & & & & & \\
\hline None & 1.0 & & & 1.0 & & & 1.0 & & \\
\hline Yes & - & - & - & - & - & - & 1.023 & 1.010 & 1.035 \\
\hline Previous abdomin & & & & & & & & & \\
\hline None & 1.0 & & & & & & 1.0 & & \\
\hline Yes & - & - & - & 1.0 & - & - & 1.254 & 0.936 & 1.578 \\
\hline Tumor node status & & & & & & & & & \\
\hline Negative & 1.0 & & & 1.0 & & & 1.0 & & \\
\hline Positive & - & - & - & - & - & - & 1.372 & 0.171 & 10.987 \\
\hline
\end{tabular}


Metcalfe et al.

disease, and some practices are simply extrapolated from the management of bladder cancer. There are no Canadian guidelines for UTUC, although the NCCN and EAU have guidelines. Unfortunately, neither guideline makes strong recommendations for specific treatment paradigms due to the lack of evidence. An analysis of practice patterns in Canada could, in principle, uncover deficiencies in current practice, but a practice can only be deemed deficient if there is clearly a better alternative. This cannot be claimed for most interventions related to UTUC. The high variability in management of UTUC in Canada underlines the urgent need for more multi-institutional controlled trials which may aid in the development of specific best practice guidelines.

The strength of this study is that it represents a broad spectrum of practice across many geographical regions. The data is Canadian and can be directly applied to our practices and patient populations. It is multi-institutional and includes a large number of patients. Limitations in this study include its retrospective design and the potential for inconsistencies in data entry between centres. Since all contributing centres in this study are academic institutions, the results do not necessarily reflect community practice.

\section{Conclusion}

There is significant variability in practice patterns for UTUC across Canada. However, when accounting for demographic, clinical and pathological variables, there was no significant difference seen in clinical outcomes that could be accounted for by the variability in practice patterns. We believe that the variability in practice patterns is primarily a reflection of the lack of treatment standards for UTUC due to the relative paucity in clinical evidence demonstrating the superiority of one practice over another. More large-scale controlled trials are required for UTUC to improve patient care.

Acknowledgements: The authors acknowledge Dr. Hassan Behlouli for assistance with statistical analysis and the Division of Urology at McGill University for funding of this project.

\section{References}

1. Siegel R, Ward E, Brawley 0 , et al. Cancer statistics, 2011: the impact of eliminating socioeconomic and racial disparities on premature cancer deaths. CA Cancer J Clin 2011;61:212-36.

2. Scher H, Bahnson R, Cohen S, et al. NCCN urothelial cancer practice guidelines. National Comprehensive Cancer Network. Oncology. Williston Park, NY;1998:225-71.

3. Roupret M, Zigeuner R, Palou J, et al. European Guidelines for the Diagnosis and Management of Upper Urinary Tract Urothelial Cell Carcinomas: 2011 Update. Actas Urol Esp 2012;36:2-14. Epub 2011 Oct 29.

4. Kamihira 0, Hattori R, Yamaguchi A, et al. Laparoscopic radical nephroureterectomy: a multicenter analysis in Japan. Eur Urol 2009;55:1397-407.

5. Manabe D, Saika T, Ebara S, et al. Comparative study of oncologic outcome of laparoscopic nephroureterectomy and standard nephroureterectomy for upper urinary tract transitional cell carcinoma. Urology 2007;69:457-61.

6. Favarefto RL, Shariat SF, Chade DC, et al. Comparison between laparoscopic and open radical nephroureterectomy in a contemporary group of patients: are recurrence and disease-specific survival associated with surgical technique? Eur Urol 2010;58:645-51.

7. Capitanio U, Shariat SF, Isbarn H, et al. Comparison of oncologic outcomes for open and laparoscopic nephroureterectomy: a multi-institutional analysis of 1249 cases. Eur Urol 2009;56:1-9. http://dx.doi. org/10.1016/i.eururo.2009.03.072

8. Gore JL, Lai J, Setodji CM, et al; and Urologic Diseases in America Project. Mortality increases when radical cystectomy is delayed more than 12 weeks: results from a Surveillance, Epidemiology, and End Results-Medicare analysis. Cancer 2009;115:988-96. http://dx.doi.org/10.1002/cncr.24052

9. Waldert $M$, Remzi $M$, Klingler $H C$, et al. The oncological results of laparoscopic nephroureterectomy for upper urinary tract transitional cell cancer are equal to those of open nephroureterectomy. BJU Int 2008; 103:66-70.

10. Sundi D, Svatek RS, Margulis V, et al. Upper tract urothelial carcinoma: impact of time to surgery. Urol Oncol 2012;30:266-72. http://dx.doi.org/10.1016/i.urolonc.2010.04.002

11. Fleshner $N$, Dranitsaris $G$, Finelli $A$, et al. Surgical wait times for patients with urological cancers: a survey of Canadian surgeons. Can J Urol 2006;13(Suppl 3):3-13.

12. Kulkarni GS, Urbach DR, Austin PC, et al. Longer wait times increase overall mortality in patients with bladder cancer. J Urol 2009;182:1318-24. http://dx.doi.org/10.1016/i.juro.2009.06.041

13. Fradet $Y$, Aprikian $A$, Dranitsaris $G$, et al. Does prolonging the time to bladder cancer surgery affect long-term cancer control: a systematic review of the literature. Can I Urol 2006;13(Suppl 3):37-47

14. Canadian Surgical Wait Times (SWAT) Initiative. Consensus document: recommendations for optimal surgical wait times for patients with urological malignancies. Can J Urol 2006; 13(Suppl 3):62-4.

15. Fairey A, Kassouff W, Black P, et al. Comparison of Oncologic Outcomes for Open and Laparoscopic Radical Nephroureterectomy: Results from the Canadian Upper Tract Collaboration. BJU Int 2012;Nov 13. http:// dx.doi.org/10.1111/j.1464-410X.2012.11474.x. [Epub ahead of print].

16. Walton TJ, Novara G, Matsumoto K, et al. Oncological outcomes after laparoscopic and open radical nephroureterectomy: results from an international cohort. BJU Int 2010;108:406-12.

17. Ariane MM, Colin P, Ouzzane A, et al. Assessment of Oncologic Control Obtained After Open Versus Laparoscopic Nephroureterectomy for Upper Urinary Tract Urothelial Carcinomas (UUT-UCS): Results from a Large French Multicenter Collaborative Study. Ann Surg Oncol 2012;19:301-8. Epub 2011 Jun 21.

Correspondence: Dr. Peter C. Black, Department of Urologic Sciences, University of British Columbia, Level 6, 2775 Laurel St., Vancouver, BC V5Z 1M9; pblack@mail.ubc.ca

Competing interests: Sources of support: Division of Urology, McGill University.

This paper has been peer-reviewed. 